\title{
Benefits from adjuvant intraoperative radiotherapy treatment for gastric cancer: A meta-analysis
}

\author{
WEI-WEI YU ${ }^{1 *}$, YAN-MEI GUO ${ }^{2 *}$, QING ZHANG ${ }^{1}$ and SHEN FU ${ }^{1}$ \\ ${ }^{1}$ Department of Radiation Oncology, Shanghai Jiao Tong University Affiliated Sixth People's Hospital; \\ ${ }^{2}$ Department of Digestive Disease, The Tenth People's Hospital, Tongji University, Shanghai, P.R. China
}

Received April 1, 2014; Accepted April 29, 2014

DOI: $10.3892 / \mathrm{mco} .2014 .444$

\begin{abstract}
The benefits of adjuvant intraoperative radiotherapy (IORT) for resectable gastric cancer have been extensively studied, but data on the survival rate remains equivocal. A meta-analysis was performed of the studies involving the use of IORT for resectable gastric cancer using web-based databases. Hazard ratios (HRs) describing the impact of adjuvant IORT on the overall survival (OS) rate and locoregional control were extracted directly from the original studies or calculated from survival curves. A meta-analysis of four studies that provided OS data revealed that IORT had no significant impact on OS [HR, 0.97; 95\% confidence interval $(\mathrm{CI}), 0.75-1.26 ; \mathrm{P}=0.837]$. In the three studies testing the efficacy of IORT for OS in the subgroup of patients with stage III disease, there was a significantly improved OS (HR, 0.60; 95\% CI, 0.40-0.89; P=0.011). Significant locoregional control improvement was observed in the four studies that provided locoregional control data (HR 0.40; 95\% CI, 0.26-0.62; $\mathrm{P}<0.001)$. This meta-analysis showed a statistically significant locoregional control benefit with the addition of IORT in patients with resectable gastric cancer. In addition, the available data revealed that adjuvant IORT may provide promising results on the survival rate for the subgroup of patients with stage III disease. Further study is required to optimize the implementation of adjuvant IORT for gastric cancer with regard to patient selection and integration with systemic therapy.
\end{abstract}

Correspondence to: $\mathrm{Dr}$ Shen Fu, Department of Radiation Oncology, Shanghai Jiao Tong University Affiliated Sixth People's Hospital, 600 Yi Shan Road, Xu Hui, Shanghai 200233, P.R. China E-mail: fushen333@126.com

\section{${ }^{*}$ Contributed equally}

Key words: gastric cancer, intraoperative radiotherapy, survival rate, meta-analysis

\section{Introduction}

Despite advances in surgical techniques, the outcome of patients with locally advanced gastric cancer following surgery remains poor (1). Locoregional recurrence is the main pattern of failure in gastric cancer patients treated with complete resection $(2,3)$. Although the efficacy of postoperative chemoradiotherapy following radical surgery for locally advanced gastric cancer has been confirmed in the INT-0116 trial (4), the prognosis remains suboptimal. Theoretically, a greater radiation dose may provide a higher tumor control. However, owing to the dose-limiting surrounding tissues in the planning treatment volume, including small intestine, pancreas, bile ducts and spinal cord, the higher doses that are necessary for disease control cannot be safely delivered with conventional external-beam radiotherapy (EBRT) (5). Intraoperative radiotherapy (IORT) allows the delivery of a boost of radiation to a localized area in a single fraction without affecting the surrounding tissues (6). An IORT boost component has also been included in the context of surgical resection, adjuvant EBRT and chemotherapy, with acceptable tolerance and improved locoregional control $(7,8)$.

Although the efficacy of IORT for locally advanced gastric cancer has been previously addressed in several studies and suggests that the addition of IORT may increase the locoregional control and thereby may improve the overall survival (OS), the results from all available studies have been equivocal (9-11). To determine whether there is a benefit of IORT for resectable gastric cancer, a meta-analysis was performed of studies that focused on this topic.

\section{Materials and methods}

Search strategy and selection criteria. A bibliographical study was performed using the PubMed, Web of knowledge and Embase electronic databases. The following medical subject headings, keywords and text words were used: i) Gastric or stomach, and cancer, carcinoma or adenocarcinoma; and ii) intraoperative radiotherapy or IORT. The search included the studies that were published between January, 1990 and July, 2013. The computer search was supplemented with a manual search of the reference lists from all available review studies, primary studies, meetings abstracts and bibliographies of books, in order to identify other studies that were not found 
during the computer search. When the results of a single study were reported in more than one publication, only the most recent and complete data were included in the meta-analysis.

The potentially eligible studies were retrieved and a full-text analysis was performed. Only the studies that included OS and/or the locoregional control rate comparison between the patients with histology-proven cancer of the stomach, assigned to surgery alone (observation arm) or to surgery plus IORT (study arm) were included in the review process. EBRT and chemotherapy were administered to the patients in both arms.

Data extraction. Data were carefully extracted independently by two investigators (W.W. Yu and Y.M. Guo) according to the preferred reporting items for systematic reviews and meta-analyses (PRISMA) statement (12). The following information was extracted from each study: First author's name, year of publication, study design, number of patients, rates of dissections and endpoints reported (OS and locoregional control rates).

Hazard ratios (HRs) for the OS and locoregional control rates were extracted directly from the original studies or were estimated indirectly by reading off survival curves as suggested by Tierney et al (13). In summary, when the estimated HR and its standard error were described in the publications, these values were obtained directly; when these statistical variables were not provided explicitly in a study they were calculated directly using two of the following parameters: The confidence interval (CI) for the HR, the log-rank statistic, the P-value or the O-E statistic (difference between numbers of observed and expected events). When those data were not available, the following were studied: The total number of events, the number of patients at risk in each group and the log-rank statistic or its P-value, allowing calculation of an approximation of the HR estimate. When the only available data were in the form of graphical representations, they were calculated from Kaplan-Meier survival curves. The Kaplan-Meier curves were read by two investigators using the Engauge Digitizer 4.1 version software (Mark Mitchell, Boston, MA, USA) independently to reduce the inaccuracy in the extracted survival rates. The HRs for OS were also extracted for the patient subgroups (including patients with stage III) whenever possible.

Statistical analysis. The heterogeneity was formally investigated by means of Cochrane Q statistic and $\mathrm{I}^{2}$ statistic. For the $\mathrm{Q}$ statistic, the heterogeneity when $\mathrm{P}<0.1$ was considered to indicate a statistically significant difference. The $\mathrm{I}^{2}$ statistic, which is the proportion of the total variation among the studies that is likely to be explained by between-study heterogeneity rather than chance (14), is reported. Substantial heterogeneity exists when $\mathrm{I}^{2}>50 \%$. When the hypothesis of homogeneity was not rejected, a fixed-effects model was used. Otherwise, the random-effects model was used (15). By convention, the impact of IORT on the OS or locoregional control rates was considered to indicate a statistical significance if the $95 \% \mathrm{CI}$ for the overall HR did not overlap 1 . The evidence of publication bias was evaluated by the funnel plot with the test of Begg and Mazumdar (16) and the linear regression asymmetry test of Egger et al (17). For these analyses, $\mathrm{P}<0.05$ was considered to indicate statistically significant publication bias. All statistical
607 records identified

through database searching

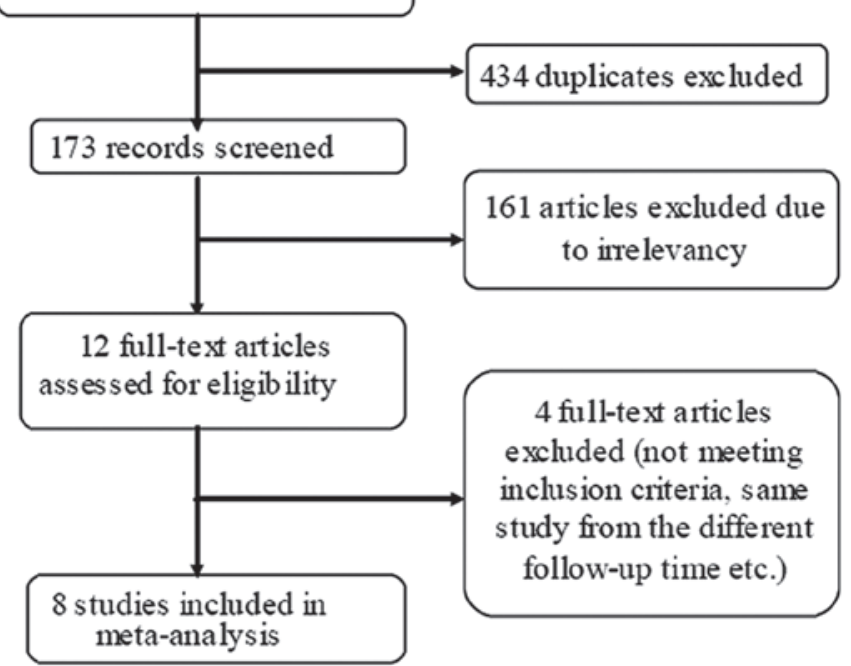

Figure 1. Flowchart of the study selection process.

analyses were performed by the STATA 12.0 software (Stata Corp., College Station, TX, USA).

\section{Results}

Trial selection and characteristics of the included studies. A total of 607 relevant studies were collected. Subsequent to the exclusion of the duplicate references by the 'find duplicates' function of EndNote X3, there were 173 unique studies. Following a review of each title and abstract, 12 studies meeting the eligibility criteria were identified. A careful examination of these full studies led to the exclusion of four studies: The updated results from one study were available in a separate publication (7) and four were excluded as they did not meet the inclusion criteria (18-20). Thus, a total of eight studies were included in the meta-analysis (8-11,21-24) (Fig. 1).

Study characteristics. The details regarding the eight studies included in the analysis are summarized in Table I. The OS data for all patients in trials were available for four studies $(8,9,11,24)$. Three studies provided the OS data for the subgroup of patients with stage III disease, and they all tested surgery followed by adjuvant IORT against surgery alone (21-23). The locoregional control rate was provided in the four studies $(8,10,11,24)$.

Meta-analysis findings. The meta-analysis of the four studies that provided data on OS revealed that IORT had no significant impact in OS. The pooled HR was 0.97 (95\% CI, 0.75-1.26; $\mathrm{Z}=0.21 ; \mathrm{P}=0.837)$, without any evidence of heterogeneity $(\mathrm{P}=0.644)$ (Fig. 2). In the three studies testing the efficacy of IORT for OS in the subgroup of patients with stage III disease, there was a significantly improved OS (HR, 0.60; 95\% CI; 0.40-0.89; $\mathrm{Z}=2.53 ; \mathrm{P}=0.011$ ) (Fig. 3). The significant locoregional control improvement was observed in the four studies that provided locoregional control data, and the combined HR was 0.40 (95\% CI, 0.26-0.62; $\mathrm{Z}=4.18 ; \mathrm{P}<0.001)$, without heterogeneity $(\mathrm{P}=0.516)$ (Fig. 4). For all eight studies, there 
Table I. Summary of the studies included in the meta-analysis.

\begin{tabular}{|c|c|c|c|c|c|c|}
\hline Author (Refs.) & Year & $\begin{array}{l}\text { Years of } \\
\text { accrual }\end{array}$ & Study design & $\begin{array}{c}\text { Nodal } \\
\text { dissection }\end{array}$ & $\mathrm{N}$ pts & $\begin{array}{l}\text { Endpoints } \\
\text { reported }\end{array}$ \\
\hline Drognitz et al (9) & 2008 & $\begin{array}{l}\text { February } 1991 \\
\text { to July } 2001\end{array}$ & $\begin{array}{l}\mathrm{S}+\mathrm{IORT}(6-15 \mathrm{MeV}, 15-25 \mathrm{~Gy}) \\
\mathrm{S}\end{array}$ & D2 & 122 & OS \\
\hline Zhang et al (8) & 2012 & $\begin{array}{l}\text { March } 2003 \\
\text { to October } 2005\end{array}$ & $\begin{array}{l}\mathrm{S}+\mathrm{IORT}(9-16 \mathrm{MeV}, 12-15 \mathrm{~Gy})+\mathrm{EBRT}+\mathrm{CT} \\
\mathrm{S}+\mathrm{EBRT}+\mathrm{CT}\end{array}$ & D2 & 97 & $\begin{array}{l}\text { OS } \\
\text { Locoregional control }\end{array}$ \\
\hline $\begin{array}{l}\text { Martinez Monge } \\
\text { et al (11) }\end{array}$ & 1997 & $\begin{array}{l}\text { October } 1982 \\
\text { to March } 1993\end{array}$ & $\begin{array}{l}\mathrm{S}+\mathrm{IORT}(9-20 \mathrm{MeV}, 10-17 \mathrm{~Gy})+\mathrm{EBRT} \\
\mathrm{S}+\mathrm{EBRT}\end{array}$ & D2 & 62 & $\begin{array}{l}\text { OS } \\
\text { Locoregional control }\end{array}$ \\
\hline Sindelar et al (24) & 1993 & No reported & $\begin{array}{l}\mathrm{S}+\operatorname{IORT}(11-15 \mathrm{MeV}, 20 \mathrm{~Gy})+\mathrm{EBRT} \\
\mathrm{S}+\mathrm{EBRT}\end{array}$ & No reported & 41 & $\begin{array}{l}\text { OS } \\
\text { Locoregional control }\end{array}$ \\
\hline Santoro et al (10) & 1998 & $\begin{array}{l}\text { July } 1976 \\
\text { to July } 1993\end{array}$ & $\begin{array}{l}\text { S + IORT (27-30 Gy) } \\
\text { S }\end{array}$ & D2 & 59 & Locoregional control \\
\hline Qin et al (21) & 2006 & 1992 to 1998 & $\begin{array}{l}\mathrm{S}+\mathrm{IORT}(6-16 \mathrm{MeV}, 10-30 \mathrm{~Gy}) \\
\mathrm{S}\end{array}$ & $\mathrm{D} 2$ or $\mathrm{D} 3$ & 292 & OS (stage III) \\
\hline Ogata et al (22) & 1995 & $\begin{array}{l}\text { August } 1983 \\
\text { to July } 1992\end{array}$ & $\begin{array}{l}\mathrm{S}+\mathrm{IORT}(12 \mathrm{MeV}, 28-30 \mathrm{~Gy}) \\
\mathrm{S}\end{array}$ & D2 & 47 & OS (stage III) \\
\hline Abe et al (23) & 1995 & No reported & $\begin{array}{l}\text { S + IORT (28-35 Gy) } \\
\text { S }\end{array}$ & No reported & 77 & OS (stage III) \\
\hline
\end{tabular}

S, surgery; OS, overall survival; EBRT, external-beam radiotherapy; IORT, intraoperative radiation therapy; N pts, number of patients; CT, chemotherapy.

\begin{tabular}{|c|c|c|}
\hline Study ID & $\mathrm{HR}(95 \% \mathrm{Cl})$ & Weight $\%$ \\
\hline zhang Q (2012) & $0.83(0.53,1.29)$ & 34.59 \\
\hline Drognitz O (2008) & $1.00(0.67,1.49)$ & 42.84 \\
\hline MartinezMonge R (1997) & $1.60(0.64,4.01)$ & 8.13 \\
\hline Sindelar WF (1993) & $1.00(0.50,1.98)$ & 14.45 \\
\hline Overall $(\mathrm{I}-$ squared $=0.0 \%, p=0.644)$ & $0.97(0.75,1.26)$ & 100.00 \\
\hline
\end{tabular}

Figure 2. Fixed-effects meta-analysis of the impact of adjuvant IORT on the overall survival rate for the entire patients in the four eligible studies. HR, hazard ratio; CI, confidence interval; IORT, intraoperative radiotherapy; EBRT, external-beam radiotherapy; CRT, chemoradiation; S, surgery.

was no evidence found of publication bias using the Egger's test $(\mathrm{P}=0.969)$ and Begg's test $(\mathrm{P}=1.0)$. The shape of the funnel plot for the pooled HR appeared to be symmetrical (Fig. 5).

\section{Discussion}

To the best of our knowledge, the present study represents the first specific meta-analysis examining the impact of IORT for patients with resectable gastric carcinoma. Although meta-analysis based on individual data is considered to be the gold standard, a meta-analysis based on the studies was still used in the present study, as individual patient data were difficult to access in the various studies published over a 20 -year period. By aggregating data from four eligible studies, it was found that the use of IORT had no significant affect on OS. Notably, when the focus was on the subgroup of patients with stage III disease, it was found that the use of IORT was associated with a clear reduction in the risk of mortality from any cause. Furthermore, the combined HR for the four eligible studies that provided locoregional control data suggested that the use of IORT was associated with a significant improvement in the locoregional control.

It is well known that local control by radiation for subclinical disease is a function of radiation dose (25). Thus, using a greater biological-radiotherapy dose could further improve the locoregional control of gastric cancer following complete surgical resection (26). However, the radiation dose to the intra-abdominal structures is usually limited to 45 Gy due to the adjacent dose-limiting structures. However, this dose may not be sufficient for the eradication of the subclinical residual disease (27). Significantly increasing the EBRT dose to the surgical bed and regional nodal areas is not acceptable when using conventional radiotherapy. IORT involves the administration of large single doses of radiation directly to surgically 


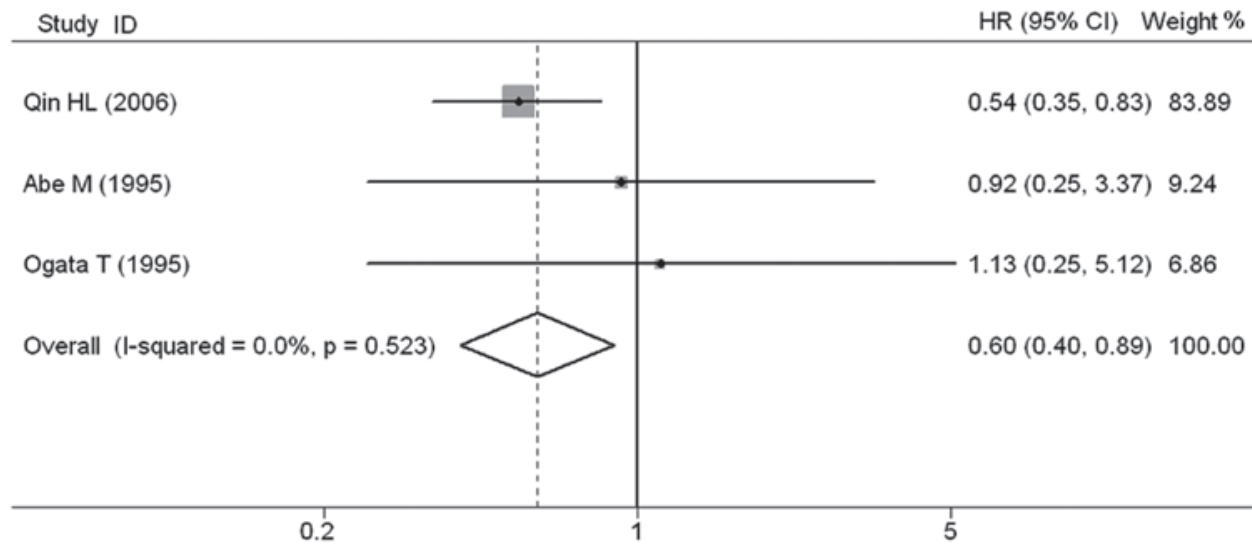

Figure 3. Fixed-effects meta-analysis of the impact of adjuvant IORT on the overall survival rate for the subgroup of patients with stage III disease in three eligible studies. HR, hazard ratio; CI, confidence interval; IORT, intraoperative radiotherapy; S, surgery.

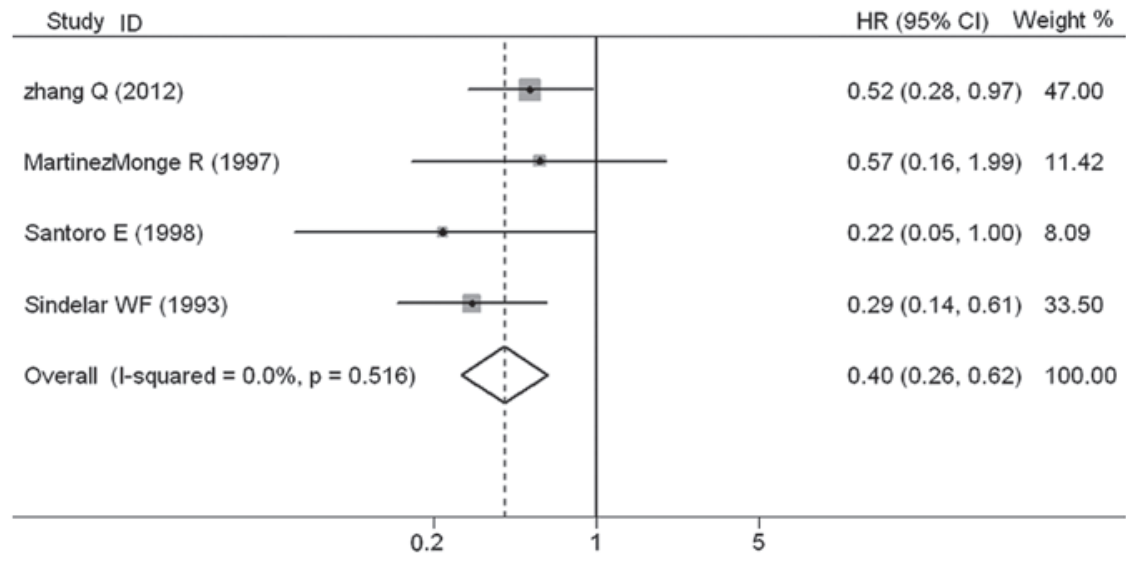

Figure 4. Fixed-effects meta-analysis of the impact of adjuvant IORT on the locoregional control rate. HR, hazard ratio; CI, confidence interval; IORT, intraoperative radiotherapy; EBRT, external-beam radiotherapy; CRT, chemoradiation; S, surgery.

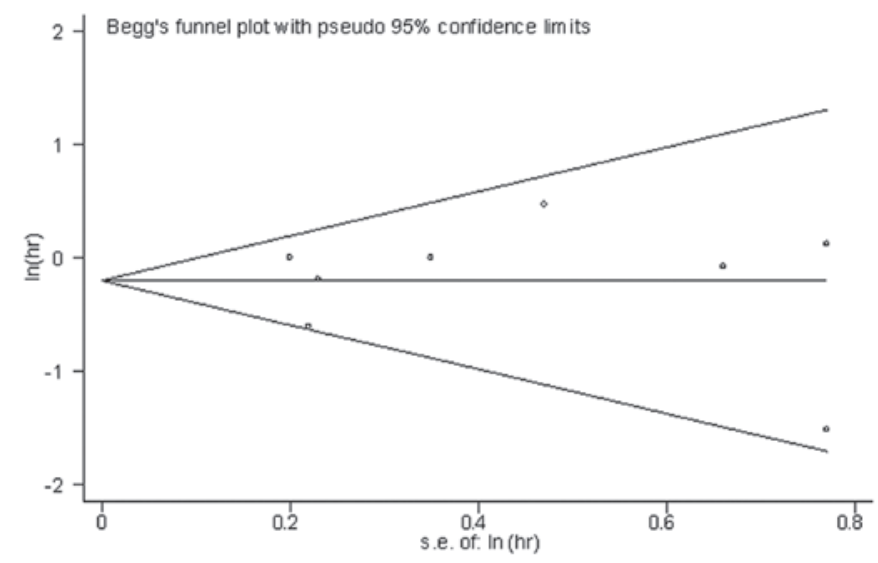

Figure 5. Begg's funnel plot for the publication bias test of the eight eligible studies. Each point represents a separate study for the indicated association. S.E., standard error.

exposed tissues during the surgical procedures. Thereby, it offers the opportunity to deliver high doses of radiation to the primary tumor and regional nodal areas, whilst simultaneously minimizing the possibility of radiation toxicity for the surrounding normal-radiosensitive tissues. IORT has been explored in various types of malignancies, including gastric cancer, with studies focusing on the benefit for the locoregional control and survival rates (28).

The findings in the present meta-analysis confirmed that the use of IORT was associated with a notable decrease in locoregional recurrence. In addition, the impact of IORT on OS reached statistical significance in the subgroup of patients with stage III disease, demonstrating that the use of IORT for locally advanced gastric cancer may yield promising results. However, the clear improvement of locoregional control did not translate into a benefit for OS in the entire cohort of patients. This may be due to the distant metastases offsetting the efficacy of IORT, emphasizing the requirement for more effective systemic therapies $(8,29,30)$. The advantage of IORT in OS may also be abolished by an increased perioperative mortality rate. Although the majority of studies did not investigate the impact of IORT on surgical complications, certain studies found an increase in the perioperative complications in conjunction with IORT $(10,20)$. The surgical and radiotherapy techniques have improved over the last twenty years, and the higher mortality rate due to adjuvant IORT-associated toxicities most probably offset the benefit of IORT.

Particular limitations of the present meta-analysis should be taken into consideration when interpreting the findings. First, the meta-analysis was performed using study-level data. 
Patient-level data, if available, may provide more reliable findings. Although there was no statistical evidence of publication bias detected, this affect cannot be ruled out. Second, numerous studies that were selected for the meta-analysis were retrospective studies, which will inevitably have had selection bias. Third, the analysis was restricted to the published studies that were written in English, and several studies that met the eligibility criteria were excluded based on language criteria. Thus, the number of eligible studies was not sufficiently large for a comprehensive analysis.

In conclusion, the present meta-analysis suggests that the use of IORT for patients with resectable gastric cancer contributed to an increase in the locoregional control rate, but did not increase the OS rate. Further study is required to optimize the implementation of adjuvant IORT for gastric cancer with regard to patient selection and integration with systemic therapy.

\section{Acknowledgements}

The present study was funded by a research grant from the Scientific Research Found Projects of Shanghai Health Bureau (grant no. 20124246) and a research grant from the National Nature Science Foundation of China (grant no. 81201883).

\section{References}

1. Hundahl SA, Phillips JL and Menck HR: The National Cancer Data Base Report on poor survival of U.S. gastric carcinoma patients treated with gastrectomy: Fifth Edition American Joint Committee on Cancer staging, proximal disease, and the 'different disease' hypothesis. Cancer 88: 921-932, 2000.

2. Gunderson LL: Gastric cancer - patterns of relapse after surgical resection. Semin Radiat Oncol 12: 150-161, 2002.

3. Oehler C and Ciernik IF: Radiation therapy and combined modality treatment of gastrointestinal carcinomas. Cancer Treat Rev 32: 119-138, 2006.

4. Macdonald JS, Smalley SR, Benedetti J, et al: Chemoradiotherapy after surgery compared with surgery alone for adenocarcinoma of the stomach or gastroesophageal junction. N Engl J Med 345: 725-730, 2001

5. Scaife CL, Calvo FA and Noyes RD: Intraoperative radiotherapy in the multimodality approach to gastric cancer. Surg Oncol Clin North Am 12: 955-964, 2003.

6. Calvo FA, Sole CV, Obregón R, et al: Intraoperative radiotherapy for the treatment of resectable locally advanced gastric adenocarcinoma: topography of locoregional recurrences and long-term outcomes. Clin Transl Oncol 15: 443-449, 2013.

7. Fu S, Lu JJ, Zhang Q, Yang Z, Peng L and Xiong F: Intraoperative radiotherapy combined with adjuvant chemoradiotherapy for locally advanced gastric adenocarcinoma. Int J Radiat Oncol Biol Phys 72: 1488-1494, 2008.

8. Zhang Q, Tey J, Peng LH, et al: Adjuvant chemoradiotherapy with or without intraoperative radiotherapy for the treatment of resectable locally advanced gastric adenocarcinoma. Radiother Oncol 102: 51-55, 2012.

9. Drognitz O, Henne K, Weissenberger C, et al: Long-term results after intraoperative radiation therapy for gastric cancer. Int $J$ Radiat Oncol Biol Phys 70: 715-721, 2008.
10. Santoro E, Carlini M, Garofalo A, Carboni F, Santoro R and Castelli M: Gastric cancer. Clinico-biological updating and analysis of 400 operated cases. J Exp Clin Cancer Res 17: 175-185, 1998.

11. Martínez-Monge R, Calvo FA, Azinovic I, et al: Patterns of failure and long-term results in high-risk resected gastric cancer treated with postoperative radiotherapy with or without intraoperative electron boost. J Surg Oncol 66: 24-29, 1997.

12. Moher D, Liberati A, Tetzlaff J and Altman DG; PRISMA Group: Preferred reporting items for systematic reviews and meta-analyses: the PRISMA statement. BMJ 339: b2535, 2009.

13. Tierney JF, Stewart LA, Ghersi D, Burdett S and Sydes MR: Practical methods for incorporating summary time-to-event data into meta-analysis. Trials 8: 16, 2007.

14. Higgins JP, Thompson SG, Deeks JJ and Altman DG: Measuring inconsistency in meta-analyses. BMJ 327: 557-560, 2003.

15. DerSimonian R and Laird N: Meta-analysis in clinical trials. Control Clin Trials 7: 177-188, 1986

16. Begg CB and Mazumdar M: Operating characteristics of a rank correlation test for publication bias. Biometrics 50: 1088-1101, 1994.

17. Egger M, Davey Smith G, Schneider M and Minder C: Bias in meta-analysis detected by a simple, graphical test. BMJ 315: 629-634, 1997

18. Henning GT, Schild SE, Stafford SL, et al: Results of irradiation or chemoirradiation for primary unresectable, locally recurrent, or grossly incomplete resection of gastric adenocarcinoma. Int J Radiat Oncol Biol Phys 46: 109-118, 2000.

19. Coquard R, Ayzac L, Gilly FN, et al: Intraoperative radiation therapy combined with limited lymph node resection in gastric cancer: an alternative to extended dissection? Int J Radiat Oncol Biol Phys 39: 1093-1098, 1997.

20. Skoropad VY, Berdov BA, Mardynski YS and Titova LN: A prospective, randomized trial of pre-operative and intraoperative radiotherapy versus surgery alone in resectable gastric cancer. Eur J Surg Oncol 26: 773-779, 2000.

21. Qin HL, Lin CH and Zhang XL: Evaluation of intraoperative radiotherapy for gastric carcinoma with D2 and D3 surgical resection. World J Gastroenterol 12: 7033-7037, 2006.

22. Ogata T, Araki K, Matsuura K, et al: A 10-year experience of intraoperative radiotherapy for gastric carcinoma and a new surgical method of creating a wider irradiation field for cases of total gastrectomy patients. Int J Radiat Oncol Biol Phys 32: 341-347, 1995.

23. Abe M, Nishimura Y and Shibamoto Y: Intraoperative radiation therapy for gastric cancer. World J Surg 19: 544-547, 1995.

24. Sindelar WF, Kinsella TJ, Tepper JE, et al: Randomized trial of intraoperative radiotherapy in carcinoma of the stomach. Am J Surg 165: 178-187, 1993.

25. Fletcher GH: Clinical dose-response curves of human malignant epithelial tumours. Br J Radiol 46: 1-12, 1973.

26. Abe M, Takahashi M, Ono K, Tobe T and Inamoto T: Japan gastric trials in intraoperative radiation therapy. Int J Radiat Oncol Biol Phys 15: 1431-1433, 1988.

27. Withers HR, Peters LJ and Taylor JM: Dose-response relationship for radiation therapy of subclinical disease. Int J Radiat Oncol Biol Phys 31: 353-359, 1995.

28. Skandarajah AR, Lynch AC, Mackay JR, Ngan S and Heriot AG: The role of intraoperative radiotherapy in solid tumors. Ann Surg Oncol 16: 735-744, 2009.

29. Miller RC, Haddock MG, Gunderson LL, et al: Intraoperative radiotherapy for treatment of locally advanced and recurrent esophageal and gastric adenocarcinomas. Dis Esophagus 19: 487-495, 2006

30. Calvo FA, Aristu JJ, Azinovic I, et al: Intraoperative and external radiotherapy in resected gastric cancer: updated report of a phase II trial. Int J Radiat Oncol Biol Phys 24: 729-736, 1992. 